\title{
The COnCEPT Project: Decoding the IT Needs of the Professional Designer When in Remote Collaboration during the Early Stages of the Design Process
}

\author{
Liapis Aggelos ${ }^{1 *}$, Didaskalou Alexandros ${ }^{2}$, Thiveos Konstantinos ${ }^{1}$ \\ ${ }^{1}$ INTRASOFT International SA, Research and Innovation Department, 19 km Markopoulou-Peania Avenue, \\ GR-19002 Peania, Athens, Greece. \\ ${ }^{2}$ Designlab Garyttou st. 150, Athens, Greece. \\ * Corresponding author. Tel.: (+30) 210 6876403; email: Aggelos.liapis@intrasoft-intl.com \\ Manuscript submitted November 5, 2014; accepted June 25, 2015. \\ doi: 10.17706/ijcce.2016.5.2.141-150
}

\begin{abstract}
Designers are frequently challenged by complex projects in which the problem space is unique, rapidly changing, and the information available is limited. In such cases, combining knowledge from different fields of expertise is required. Furthermore, collaboration during the design process is essential for achieving a meaningful and well-formed solution. Designers therefore regularly find themselves exchanging ideas and reflections in form of emails, sketches, and images with a group of experts from different backgrounds, working altogether through the creation of a design, its development and proper implementation. The particular paper explores -through COnCEPT research and development EU co-funded project- the nature and requirements of early design work focusing especially on issues of synchronous and asynchronous collaboration, team dynamics and the management and monitoring of the early stages of the design process. The overall aim is to identify the essential characteristics and needs of distributed teams when in remote collaboration during the early stages of the design process and suggest a prototype environment based on their requirements and workflow.
\end{abstract}

Key words: Brainstorming, creativity, conceptual design, CSCW, design process.

\section{Introduction}

The working activities of design teams are supported by different collaboration tools on many levels communication, sharing documents, exchanging images, transferring media files, organising tasks, time-tracking, managing the progress of a project and "monitoring" work of team mates. The technology itself creates opportunities and imposes limitations and constraints while trying to mirror, mediate or augment the natural communication between team members. However, it can be the case that digital tools designed without attention to end-user requirements can even hinder designers rather than supporting the goal of the project at hand [1], [2]. The biggest challenge for the collaboration technology lies in the fact that design is a social process. It is crucial to establish a common base of understanding the problem i.e. secure a mutual definition and acceptance of the context during the problem exploration and the paths to follow for solving this problem [2]. Achieving a common understanding among all the team members would be expected to be easier when using video conference platforms, online shared repositories and other opportunities of digital communication [3]. Nevertheless, collaboration is not only about sharing 
information. To understand the significance of this specific technology, we should situate it in the context of the social field in which it is used [3], [4]. We should understand the social practices, the power relations and the points of tension between designers and other stakeholders [4]. A project might include interactions within the same organisation, between different organisations and even with mass communities of collaborators. Furthermore, these interactions could happen at geographical spaces of significantly different conditions - starting from the same room, the same floor, same building, same city, same or different country, and even across different time zones [5].

This paper explores the nature of early design work, and focuses especially on issues of collaboration and document sharing needs, aiming at identifying what are the essential characteristics of distributed creative collaboration within design teams. The reported research combines several research methods, such as: Analysis of existing tools from a user perspective; Surveys and interviews of designers regarding early design work, the tools and methods they use, with an emphasis on collaboration; Case study analysis of eight (8) design projects conducted in UK and Greece; An experimental evaluation of a tool to support early design.

\section{Experiential Design Sessions}

The research described the usage in design of some state of the art tools currently used by designers. During each experiential session, the evaluation of a specific tool was done with the goal to identify points of improvement. The findings from this section should go beyond usability evaluation and ultimately should be incorporated in the list of requirements of the COnCEPT platform.

\subsection{Mind Mapping and Brainstorming Tools}

Mind mapping and brainstorming tools are used to create diagrams of relationships between concepts, ideas or other pieces of information. Their popular uses include project planning, collecting and organising thoughts; brainstorming and presentations - all in order to help solve problems, map out resources and uncover new ideas. Some of the properties of mind maps that have to be supported in order to attest the effectiveness of mind mapping include [5]:

- Keyword Orientation: the structural elements of mind maps are not sentences but keywords.

- Loose Syntax and Semantics: association is the only relationship between linked keywords.

- High-Level View: overview of a whole mind map in a glance.

- Evocative: a mind map evokes the context of the scene in which it was created.

- Semi-structured: a mind map can have a template structure but it can grow branches on demand to capture real-time verbal communication in semi-structured interview.

There are various tools available for providing mind mapping functionalities, most of which are web applications, making it even easier to use them anywhere from any web browser [6]. Based on a wide search on the available tools, it was concluded that the most prominent tools in this category to be used by product designers within COnCEPT are FreeMind, Coggle and WiseMapping.

\subsection{Sketching and Storyboarding Tools}

With storyboarding, the designer turns ideas and goals of a projected user experience into something visual. In this way ideas are easier for other people to understand, and to give constructive feedback. Ideas are brought to life with storyboard shapes, text, animations, and all the other features that PowerPoint Storyboarding provides [7]. The following properties have to be supported by storyboarding tools:

- Editing and Re-use: the designer must often redraw features that have not changed. In order to avoid such repetitions, a manual translation to an electronic format is required;

- Design Memory: the sketches may be annotated, but a designer cannot easily search these annotations 
in the future to find out why a particular design decision was made. Practicing designers have found that the annotations of design sketches serve as a diary of the design process, which are often more valuable to the client than the sketches themselves.

- Interactivity: interaction between the concept and the user has to be supported. In order to actually see what the interaction would be like, a designer needs to "play with the computer" and manipulate several sketches in response to a user's verbalised actions. Designers need tools that give them the freedom to quickly sketch rough design ideas and to test the designs by interacting with them.

\subsection{Conceptual Modelling Tools}

The term conceptual model may be used to refer to models which are formed after a conceptualisation process in the mind. Conceptual models represent human intentions or semantics. Conceptualisation from observation of physical existence and conceptual modelling are the necessary means humans employ to think and solve problems. Concepts are used to convey semantics during various natural languages based communication [7]. Since a concept might map to multiple semantics by itself, an explicit formalisation is usually required for identifying and locating the intended semantic from several candidates to avoid misunderstandings and confusions in conceptual models.

Within COnCEPT, conceptual modelling tools are envisaged to be used from various perspectives, serving a range of applications, from conceptual modelling of interactions within a process up to conceptual modelling of the designed ontologies.

\section{Web Survey: Collaboration Practice}

The web survey consisted of 32 questions and concerned (1) the design practitioner, (2) the design team, (3) artefacts and documents created and used during design activities, (4) media and devices used during design activities, (5) skills and difficulties that are faced during design activities and (6) general information of the respondent. The survey was launched online on 23 December 2013. Respondents were invited through different international channels (mailing lists) for human-computer interaction, web design, design practitioners and schools of design.

\subsection{Results}

The survey resulted in 82 responses; 32 female and 50 male respondents of an age ranged from 21 to 56 or older. The respondents were located in 16 different countries: 58 respondents currently reside in Europe, and the others reside in the United States, Canada, Australia and Asia. The professional roles of the respondents cover a wide range of design disciplines such as user interface design, user experience design or interaction design. 68 respondents are involved in one or more of these types of design, while 14 respondents are not involved in user interface design, user-experience design or interaction design. 29 respondents are solely involved in user interface design, user experience design or interaction design, while 53 respondents are (also) involved in design disciplines other than user interface design, user experience design or interaction design.

When asking about the approaches to exchange information in design projects, several approaches were considered, including real time face-to-face meetings, the use of a war room/design studio, video conference, teleconference, chat/instant messaging/social media, e-mail, wiki/blog and cloud-based documentation sharing services. E-mail is the approach which is used most of the time or often for information exchange by the majority of the respondents. Next, real time face-to-face meetings and cloud based documentation are also frequently used by the majority of the respondents.

An open question, inquired the respondents regarding the specific problems they have to deal with in 
their projects. Table 1 presents the most common problems mentioned by the respondents. Other problems mentioned, are "budget and timing".

Table 1. Most Common Problems in Collaborative Design

\begin{tabular}{|l|l|l|}
\hline & Collaborative creation of designs & Informing team members and other people involved \\
\hline $\begin{array}{l}\text { Communication } \\
\text { problems }\end{array}$ & $\begin{array}{l}\text { "the status of progress", remote } \\
\text { communication / "pointing to something is } \\
\text { an issue if we do not share the same space", } \\
\text { "communication of design decisions", } \\
\text { "different backgrounds" }\end{array}$ & $\begin{array}{l}\text { "choosing best medium", "design misinterpreted", "hard } \\
\text { to convey design process", "language issues", "people } \\
\text { interpret what they hear in different ways", "late } \\
\text { feedback" }\end{array}$ \\
\hline $\begin{array}{l}\text { Technical } \\
\text { problems }\end{array}$ & $\begin{array}{l}\text { "versioning / tracking changes", "different } \\
\text { platforms", "cannot represent everything on } \\
\text { a whiteboard, especially when it involves } \\
\text { dynamics/animations", "difficult to 'create' } \\
\text { and 'brainstorm' while in separate } \\
\text { locations" }\end{array}$ & $\begin{array}{l}\text { "compatibility", "versioning", "phone and web sharing } \\
\text { problems", "network problems" }\end{array}$ \\
\hline
\end{tabular}

\section{First Round of Interviews with Professional Designers}

At the end of the survey, 25 respondents volunteered for follow-up interviews. The intention was to contact the volunteering respondents for further user studies enriching the initial data received from the first survey. Designers confirmed that they often use e-mail. They also strengthened that the use of the approaches depends on the type of tasks that are conducted at each moment of the project. If possible, they prefer face-to-face communication to discuss design ideas and decisions, while design elements are shared with team members by e-mail or cloud based documents sharing. The settings, used most of the time or often to collaborate and communicate with people involved in the project are at the same location, at the same time and at different locations, at the same time. Several professional designers prefer to collaborate and communicate at the same location at the same time. This also allows them to use the same tool (i.e. Illustrator) for individual work, collaboration and communication as they can use it while talking with other team members. There are an equal amount of technical problems and communication problems when the respondents create designs in collaboration, while there are less technical problems than communication problems when the respondents inform team members and people involved in the project about their design choices. One of the major problems faced during communication, is the use of different or not common terms due to the different disciplines and backgrounds within a design team, which implies difficulties to understand each other during collaboration and communication.

When considering the problems specified by the respondents, we conclude that many of the problems mentioned are linked to issues like: status of progress, communication of design decisions, choosing the best medium, design misinterpretation, versioning/tracking changes, difficulties to 'create' in separate locations and compatibility. The biggest challenges for COnCEPT were not reported as technical issues, but communication issues.

\section{Second Web Survey: Collaboration Tools}

The online survey was distributed through social networks (LinkedIn, Facebook) among 12 design-related groups in the Netherlands. 159 people (mostly indicating an age between 30 and 40 years) responded during the first two weeks of March, 2014. This response rate corresponds to $0.0015 \%$ of the total number of members ( 40632 people) that were reached through the online design groups. Out of the 159 respondents of the web survey, 42 are currently based in the Netherlands, 34 are based in the Czech Republic, 23 in Finland, 20 in UK and 12 in Belgium. Having the possibility to select multiple options, most of the respondents indicated being currently active in the disciplines of user experience design $(32,2 \%)$, 
interaction design $(27,1 \%)$, visual/graphic design $(27,1 \%)$, industrial design $(27,1 \%)$, web design $(11,9 \%)$, or interior design $(11,9 \%)$. The size of the latest team in which the respondents worked (including themselves) was 3 to 4 people in $44,1 \%$ cases, 5 to 9 people in $28,8 \%$ cases, 2 people in $11,9 \%$ cases, and a single person (i.e., only the respondent) in 10,2\% cases. The rest worked in larger than 9 member's teams. Respondents indicated that they collaborate with the team members located in a different time zone in 15,4\% of the cases, whereas $32,7 \%$ responded that they collaborate with the rest of the team within the same room, $17,3 \%$ within the same floor, $9,6 \%$ within the same building, $15,4 \%$ within the same city, $28,9 \%$ within the same country, and $7,7 \%$ within the same time zone.

A first look in the results showed that overall, 98,3\% respondents use computer at work and 94,9\% use Internet for activities related to work, both on a daily basis. These numbers indicate that the Internet through its diverse online services actively supports the work of designers. A closer look on the topic of collaboration showed that $82,7 \%$ of the respondents used several online collaboration tools to support the work of their team in the past 6 months. We asked respondents of the survey to explain the reasons why they liked or disliked using online collaboration tools. The positive responses included mostly properties and functionality such as: easy to use, fast, simultaneous editing, multi-platform / multi device, accessible from anywhere, drag-n-drop functionality, chat, history tracking, sharing, real-time, everything at one place, comments, alerts / notifications.

On the other hand, negative responses mentioned the following annoying properties or insufficient functionality when using online collaboration tools: complicated setup of user account required, being unstable, slow, confusing, buggy, non-intuitive, creating conflicted copies, complicated user interface, mutual disturbance during simultaneous document editing. Additionally, we explored what was the relation between mobile services and team collaboration. The mobile adoption in our survey uncovered that $91,5 \%$ respondents owns a smartphone (44,1\% iPhone, 40,7\% Android phone, 6,8\% Windows Phone), and 52,5\% respondents own a tablet (37,3\% iPad, 13,6\% Android tablet). 91,4\% respondents use a smart phone or a tablet for their work (35,5\% on a daily basis, $25,9 \%$ a couple of times a week).

\section{Second Round of Interviews with Professional Designers}

From the total number of participants of the interview, 25 respondents indicated that they wanted to participate further in the research, and provided their contact information. We selected 15 participants from the web survey, based on their design specialisation. The types of specialisation of the people that were interviewed were: UI Designer, Industrial / Product Designer, student of Industrial Design, Visual and Graphic Designer, Interaction Designer, User Experience Designer, Game Designer, Industrial and UX Designer. Brainstorming is done most often together with other team members or stakeholders, typically in one room, on a paper. Pinterest and/or a shared folder with interesting materials serves as a source of inspiration. Usually, no special tools are used in the stages of creative design or idea generation; however, when tools are used, these include: UX Pin, Axure, Indigo Studio, Adobe Ideas, Mind mapping tool of Google Drive, and sketching tools such as Paper by 53.

Designers use most frequently Dropbox and Google Drive for sharing documents and various multimedia files. The Intranet shared repository is used frequently too, especially in case of confidential projects that cannot be shared on available public services. Pinterest is often used for sharing images, especially for inspiration (library of characters, scenes, UI elements, etc.), while code repositories like SVN, Bitbucket, or SourceTree are also used. Finally, files or URLs of the files are usually shared via email. Tasks are typically divided during group meetings, and one person (project manager / team leader) is usually responsible for the final decision at every process stage. Tools used for assigning tasks and tracking the process, include: Trello, Teambox, or Excel. Face-to-face meetings with other team members do not need to take place on a 
daily or weekly basis, sometimes they occure as rarely as on a monthly basis. However, organising time is done often individually, and is closely related to the task organisation. Some companies use Gantt charts (using online tools, such as Redmine), or shared calendars to keep track of the availability and the progress of the others. Companies use various tools for communication, mostly Skype, WhatsApp, and Facebook (groups) due to their advantage of real-time communication and immediate response. For non-real-time communication, email is a standard way of sending documents to other team members. Text messages or phone calls are preferred in cases of emergency. Communication with clients is done mostly via email and Skype, and often includes URLs of interactive prototypes or other deliverables that can be commented directly (in cases where the tool allows it - e.g., comments in Google Drive documents, etc.), or indirectly (taking a screenshot and writing comments on it, etc.). Clients typically do not communicate with designers on a daily basis - communication is usually mediated by the team leader or project manager, who filters, discusses, and/or prioritises the information before passing it to the rest of the team. Face to face contact is mostly preferred.

Problems are also often about tools that cannot be installed or accessed due to internal company network security policies, and tools that are not intuitive enough for the team members or clients to easily embrace . These cases are often solved in very inconvenient ways, such as printing out the documents, writing comments on them, scanning them again and sending back to the other side, instead of using some direct, online commenting functionality. Another example of problem is the involuntary creation of conflicting copies, when people work simultaneously on the same file. Keeping time-schedules, tasks, and project progress up-to-date is yet another common problem. Last but not least, using communication tools that include contacts which are not related to the work environment, is often distracting.

\section{COnCEPT Platform Reference Framework}

Based on the results of the survey, the COnCEPT project released a high level framework of the platform, which clearly identifies the high-level components of the system, and the relationships among them. Its purpose is to direct attention at an appropriate abstraction of the system without delving into details [1]-[3], [8], [9]. The design and deployment of the COnCEPT Architecture is targeted at the support of a set of functionalities, based on the designers' needs identified from the analysis of the surveys and interviews. The phases for the deployment of projects include the creation of new projects (as well as the management of existing ones), the Brief Analysis of the projects combined with the initial brainstorming phase, the collection of a set of ideas and materials in a MoodBoard, the generation of a StoryBoard incorporating a set of design concepts, taking into account existing material and assisting the selection process of the desired concept, the Sketching of the concept, and finally the presentation of the final product to the client. Within all phases, proper logging of the activities of the team members and support for addition of annotations, when applicable, will be supported. Furthermore, proper interconnection interfaces will be designed and implemented for the proper interconnection of the diverse tools deployed in the platform, while the main platform will be based on the installation and configuration of a Content Management System (CMS).

Upon finalization of each phase of the design process, the produced outcomes may be communicated to the client for receiving feedback, offering the option for comments as well as providing confirmation functionality for advancing to the next phase. In the following paragraphs, the different phases of the COnCEPT Design Process are shortly described and associated with the components that will be deployed.

Create a new project - Design Brief: In this phase, the initiation of new project as well as the management of existing ones will be supported. A custom software component will be developed accompanied with proper user interfaces. The end user is going to provide details and specifications describing essential project components: the design brief, the client assigning the project, the cost model to be followed, details 
regarding the contract, project team members' selection, role and tasks assignment etc.

Brief Analysis - Decoding process: In this phase, a detailed description of the project will be provided and accompanied with text and metadata analysis, further addition of metadata as well as suggestions to end users. Text analysis will be realized based on Named-Entity Recognition (NER) techniques while the text may be in word, pdf or plain text format. Suggestions based on the interconnection with existing free web dictionaries as well as the association of recognized entities with relevant entities in the Linked Open Data (LoD) Cloud will be supported. Searching functionalities for relevant resources based on the initial analysis and parameters of the documents will be also available (this can be done in local database or web sources).

Brainstorming process: In this phase, brainstorming functionalities will be supported based on the use of components available via the CMS. These components include modules for realization of mindmaps as well as modules for adding post-it-notes. Based on the text provided by the end user, automatic suggestions for extensions will be provided. The extensions will be based on the description of the associated concepts in the COnCEPT Ontology (description of concepts per design domain). In the post-it-notes module, addition of text and images will be supported.

Moodboards: In this phase, collection of material in parallel with addition of ideas/concepts from the designer will be collected on a whiteboard. Sketching functionality will be supported for initial sketching of ideas, while links to the material already available from the brainstorming process will be included. Interconnection with a visual search engine (search for keywords, images, sketches) will be supported. Sharing functionalities of the provided/developed material among the team members will be also included. Collaboration in this phase will be realized in an asynchronous way.

StoryBoard: In this phase, the developed component will support the design of a set of concepts targeting the selection of the best one based on the project's requirements. Each concept will be associated with a set of slides depicting details regarding the product in the format of a storyboard. Synchronous collaboration among the designers will be supported while a toolbar will be available providing access to material developed in the previous phases. Sharing with the team as well as the client will be also supported.

Sketching: In this phase, the selected concept from the storyboard will be used as a basis for proceeding to sketching of the product. Input from the moodboard will be also used, including suggestions based on the annotated content. Collaborative sketching characteristics will be included, while export functionalities of the final product to re-usable image formats will be supported.

Concept Modeling Tools and COnCEPT Ontology: a tool for model creation will be deployed that will permit the integration and extension of existing ontologies per design domain. User friendly editing interfaces will be provided.

Annotation Mechanisms: Annotation of text and images will be supported mainly during the first phases of the COnCEPT design process (Brief Analysis and Moodboard).

\section{Research Limitations}

COnCEPT is by itself a standalone web-based platform, which will allow professional designers to collaborate during the early stages of the design process. Furthermore, COnCEPT will assist in the management of the various stages of the design process. Such large scale system contains many fine points that first need to be fully optimized in order to maximize its benefit to the creative industries, especially focusing to product designers. Specific care must also be given to the cultural and ethical issues arising when confidentiality, trust, security and IPR are involved, in order to assist professional designers to maintain awareness while promoting creativity.

\section{Conclusions}

Based on the examination of a set of collaborative tools for mind mapping and brainstorming, sketching 
and storyboarding tools as well as conceptual modeling tools, it can be claimed that within COnCEPT a diverse set of functionalities are going to be provided. Most of the tools that will be used for the implementation of the overall COnCEPT platform will be envisaged to be open source as well as provide open APIs for the design and deployment of advanced functionalities. The collaborative tools that will be deployed will support heterogeneous input and output formats, facilitate the collaborative editing and updating of information, support the tracking of changes/additions from different end-users as well as the addition of different types of annotations and will provide options for increased interactivity and exchange of knowledge among the involved users. The tools will be integrated in a unified platform -based on the installation of a dynamic content management system- and proper interfaces for their interconnection will be developed and implemented.

This survey revealed some interesting insights regarding designers and the way they collaborate and communicate with team members and other people involved in design projects. At the end of the analysis of this study, we identified a list of opportunities that should be brought into discussion while formulating the list of user requirements regarding a collaboration tool:

1) Carefully consider which (collaborative) tasks conducted by the design team and stages in the design project can be supported by features of the COnCEPT tool.

2) The need of a tool that tracks all design decisions, the progress of the project etc.

3) It is important for COnCEPT to consider the difference between design tools and communication tools. It is likely that the best choice will be including a set of (existing) design tools balanced by supporting common communication channels.

4) The biggest challenges for COnCEPT are not technical issues, but communication issues. By considering suitable ways to support communication within the design team, COnCEPT can contribute to improved collaboration efficiency within design teams.

Surprisingly, the results from the survey presented that social media sites were the most frequently referred tools for supporting collaboration. Tools that supported instant communication and file sharing were the second and third most referred collaboration technologies. Online edition of documents and integrated collaboration platforms appeared to be widely used. Finally, email and task tracking (project management) services were other recurrent tools. While each cluster of tools provides different core functionalities, (e.g. social media vs. instant communication), some of them have similar if not exact overlapping features. For instance, Facebook provides individual and group instant messaging. Another example is Google Docs, which permits the online edition of documents, and includes the functionality to chat with all the individuals that are editing the document at the same moment. A more detailed discussion about the specific tools should be centered in the context and the activities in which they are used. In extraordinary cases, users may even use one tool to accomplish a completely different task than the one the tool is intended for.

\section{Acknowledgements}

This work has been partially funded by the EC under the 7th Framework Programme, (ICT-2013.8.1: Technologies and scientific foundations in the field of creativity under grant agreement number FP7-ICT-2013-10-610725-COnCEPT COllaborative CrEative design PlaTform. The surveys have been implemented by University of Hasselt.

\section{References}

[1] Liapis, A. (2008). Synergy: A prototype collaborative environment to support the conceptual stages of the design process. Proceedings of International Conference on Digital Interactive Media in Entertainment and Arts. ACM Digital Library. 
[2] Liapis, A. (2014). Computer Mediated Collaborative Design Environments: Methods and Frameworks to Integrate Creative Tools to Support the Early Stages of the Design Process. LAMBERT Academic Publishing.

[3] Liapis, A., Kantorovitch, J., Malins, J., Zafeiropoulos, A., Haesen, M., Gutierrez, M., Funk, M., Alcamtara, J., Moore, J. P., and Maciver, F. (2014). COnCEPT: Developing intelligent information systems to support collaborative working across design teams. Proceedings of 9th International Joint Conference on Software Technologies. Vienna, Austria.

[4] Kung, C. H., \& Solvberg, A. (1986). Activity modeling and behavior modeling. In T. Ollie, H. Sol, \& A. Verrjin-Stuart (Eds.), Proceedings of the IFIP WG 8.1 Working Conference on Comparative Review of Information Systems Design Methodologies: Improving the Practice (pp. 145-171). Amsterdam, North-Holland.

[5] Exploring user requirements through mind mapping. From http://www.change-vision.com/en/ExploringUserRequirementsThroughMindMapping_Letter.pdf

[6] Malins, J., Liapis, A., Markopoulos, P. Laing, R., Coninx, K., Kantorovitch, J., Didaskalou, A., \& Maciver, F. (2014). Supporting the early stages of the product design process: Using an integrated collaborative environment. Proceedings of 6th International Conference on Engineering and Product Design Education Conference. University of Twente, Enschede, The Netherlands.

[7] Martens, J. B. (2012). Statistics from an HCI perspective: Illmo - Interactive log likelihood modeling. In G. Tortora, S. Levialdi, \& M. Tucci (Eds.), Proceedings of the International Working Conference on Advanced Visual Interfaces (pp. 382-385). ACM, New York, NY, USA.

[8] Ozcelik, D., Quevedo-Fernandez, J., Thalen, J., \& Terken, J. (2011). Engaging users in the early phases of the design process: Attitudes, concerns and challenges from industrial practice. Proceedings of DPPI ' 11 .

[9] Fernandez, J. Q., Ozcelik, D., \& Martens, J. B. O. S. (2013). A user-centered-design perspective on systems to support co-located design collaboration. Proceedings of HCI '13.

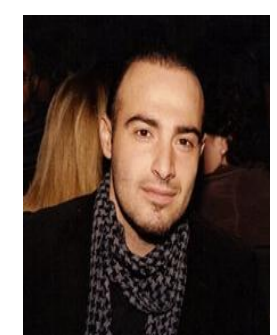

Liapis Aggelos is an experienced senior R\&D manager, active researcher and ICT specialist with experience in implementing and managing EC funded ICT projects (FP7 ICT and SEC, PSP-ICT, ENPI). His areas of interest and expertise include: CSCW, semantics, Web 2.0, e-government, e-learning, e-health, crisis management, social and cloud computing and enterprise interoperability. He has strong skills in project and quality management activities, business process analysis, preparation and maintenance of project quality plans, quality reporting, responsibility of the deliverables, preparation of project reports and coordination of consortia. He has excellent knowledge of project management methodologies and tools. Since 2004, he has worked in several relevant posts in both academia and industry, and in 2009 he was honored with the position of associate professor/senior research fellow from the Robert Gordon University in Aberdeen, Scotland.

Dr. Aggelos Liapis was a Marie Currie postdoc on the three dimensional anatomical human (3DAH) research and training network, holds a $\mathrm{PhD}$ degree in computer mediated collaborative design environments from the Robert Gordon University in Aberdeen, two masters degrees from the Universities of Sunderland and Hull in network systems and computer graphics and virtual environments, respectively, and a bachelor's degree in software development from Lincoln University in Hull. Beyond his academic and industrial duties, Dr. Liapis has authored several papers, journals and books in the areas of CSCW, computer mediated creativity, collaborative design, semantics, etc. 


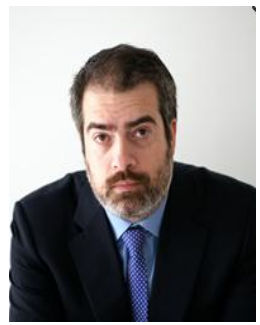

Alexandros Didaskalou is an awarded industrial designer combining scientific background with engineering skills and holds an industrial design diploma of the Hochschule für Bildende Künste Braunschweig in Germany and a master of arts degree of the Royal College of Art London. Alexandros is the CEO and founder of Designlab, an industrial design, system \& space innovation company based in Athens, Greece. Designlab is also actively participating in design research projects funded by the European Commission. Alexandros contributed his industrial design expertise to leading corporate and institutional organizations including Intrasoft, Raycap, EVIK, SAS, Terra, OBI/HIPO, Athens 2004, Diageo, ADG/Campingaz/Coleman, Cosmote, Everest, Coca-Cola Hellenic, Athens International Airport, Regency, Katselis, Resoul, Lanitis, Intracom, Coco-Mat and others. His work has been displayed at the Salone del Mobile in Milano, the RCA London, Biennale - St. Etienne, the IDF Hannover, Furnidec and several other international fairs and events. He was awarded first prizes and other distinctions at various international design competitions.

Alexandros is a $\mathrm{PhD}$ candidate at the Aristotle University of Thessaloniki, he has participated in international conferences as a design expert and has contributed to various publications about design methodology \& practices. He has been teaching industrial design \& innovation methods for the last 14 years in various vocational and educational institutions such as the Mechanical Engineering Dept. of the NTUA, AKTO, AAS and the Furniture Design Department of TEI. He participated at numerous events as a specialized speaker about product design and he is also a scientific associate of the Thessaloniki Design Museum. He speaks English, German, Italian, Spanish and Greek and also has a highest education degree in theoretical musical studies from the State Conservatory of Thessaloniki.

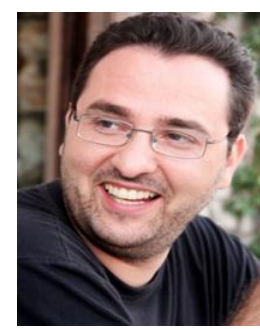

Konstantinos Thiveos holds a BSc degree in computer science from the Athens University of Economics and Business and an MSc degree in analysis, design and management of information systems from London School of Economics and Political Science. He has been working as a researcher in the Hellenic Trading Research Unit of the Athens University of Economics and Business in fields such as electronic and mobile commerce, business modeling etc. In 2001 he moved for two years in Brussels working as the Brussels Office manager of Pouliadis Group, dealing mostly with the European business expansion of the Group and the overall management of European co-funded research and development projects. He became the director of Research and Development Department of Pouliadis Associates in Athens, which had been active in various research fields and had undertaken more than 35 European and national projects. Since the end of 2005, he has been working as a senior consultant and later as a senior delivery manager in INTRASOFT International in the Research and Innovation Development (RID) Department dealing with conception and management of R\&D projects. RID is closely observing emerging ICT technologies while listening to the markets in order to conceive and develop innovative solutions that could offer a competitive market value to the company. Projects undertaken by the RID department target at assessing emerging technologies, monitoring industry innovations, seeking new market opportunities within the company's distinctive competence, initiating market research and sizing markets, and documenting needs of both traditional and emerging market sectors. Based on these insights, promising application prototypes and business cases for innovative solutions are created and validated. 